\title{
Scrotal Neoplasia: Would Truck Drivers Be At Greater Risk?
}

\author{
Daniel Seabra, Gilberto Fava, Eliney Faria, Teoclito Sacheto, Geraldo Hidalgo
}

Sections of Urology and Pathology, PIO XII Foundation, Cancer Hospital of Barretos, Barretos, Sao Paulo, Brazil

\begin{abstract}
Objective: To analyze how scrotal neoplasias have been managed during the past decade and to question possible factors or professions associated to its presence.

Materials and Methods: We retrospectively evaluated every case reported from 1995 to 2005 at our hospital. We described the clinical scenario, complementary exams, treatments and outcomes. We also tried to verify if there was any risk, predisposing factors or professions that would explain the cancer origin.

Results: Six cases were reviewed. Out of these, three patients were truck drivers. Five of them showed restricted lesions without inguinal lymph nodes enlargement. Histologically, six patients presented squamous carcinoma, with two of them having the verrucous type. The median age of patients was 52 years old (31 to 89). The five patients who are still alive had their lesions completely removed with safety margin and primary closure.

Conclusions: We have noticed that the scrotal carcinoma behavior is similar to that of the penis, where removal of the lesion and study of the regional lymph nodes help to increase the patient survival rate. The outstanding fact was that three out of six patients were truck drivers, raising the hypothesis that such profession, maybe due to the contact or attrition with the diesel exhaust expelled by the engine or to sexual promiscuity, would imply in a larger risk of developing this rare neoplasia.
\end{abstract}

Key words: urogenital neoplasms; scrotum; squamous cell carcinoma

Int Braz J Urol. 2007; 33: 515-20

\section{INTRODUCTION}

Scrotum malignant neoplasia is a rare disease and it has been occasionally reported. Its historical context is always remembered as it was initially described by Bassius, in 1731, and soon after that by Treyling, in 1740. However, in 1775, in the famous report "Cancer Scroti", Sir Percival Pott was the first one to link these tumors to chimney sweepers and, since then, this disease has been considered the first occupational neoplasia described in the medical literature. Noticing that these professionals had precarious hygiene, he advised them to take a daily bath. Soon after, the Danish association of chimney sweepers requested daily hygiene from their members, which reduced the incidence of the disease $(1,2)$. This incident was considered one of the first and the most effective interventions in Public Health. Nowadays, scrotal cancer corresponds to $0.1 / 100,000$ cases a year in the USA. Even in America, the largest oncological hospitals have, at the most, a few dozen cases in their files (3). In Brazil, it has been reported in a ship's engine operator (4). Scrotal cancer is extremely similar to penis tumors, and its management has been based on the protocols adopted for the latter. We reported the six cases seen at our hospital during 
the last decade and reviewed the literature on the subject.

We retrospectively analyzed the cases of malignant scrotal neoplasia seen in our service from 1995 to 2005 . Six cases were reported. Even if briefly, we took the time to describe the clinical scenario, diagnostic and therapeutic strategies adopted, and the evolution of every specific case. Besides, we also tried to verify if there was some risk, predisposing factor or profession that would explain the origin of this neoplasia.

\section{CASE REPORTS}

Case \#1 - B.S., 45 year-old black male, truck driver. Nine months before the initial visit, he noticed the appearance of a lesion in the scrotum, which had developed into a urethro-scrotal fistula. The patient was submitted to incisional biopsy, which demonstrated a squamous cell carcinoma. The lesion was considered irresectable and, during staging exams, the presence of bilateral inguinal lymph node and pulmonary metastasis was noticed. In July 1995, the patient was submitted to systemic radio and chemotherapy with BEP - bleomycin, etoposide, and cisplatin. The overall clinical status of the patient worsened and he died of sepsis in November 1995.

Case \#2 - J.C.S., 49 year-old black male, farmer. Ten years before the initial visit, he was submitted to perineal cutaneous urethrostomy in another hospital due to complex and recurrent stenosis of the urethra. Three months prior to coming to our hospital, he noticed a scrotal nodule, close to the urethrostomy. The patient was submitted to incisional biopsy, which was diagnosed as squamous cell carcinoma. Staging exams did not show any metastasis. In August 1998, the patient was submitted to a complete removal of the lesion, with a safety margin of $2.0 \mathrm{~cm}$. The lesion dimensions were $6.0 \mathrm{x}$ $4.0 \times 2.5 \mathrm{~cm}$. The result was compatible with the biopsy, with free margins. He progressed with stenosis of the perineal urethrostomy, which was solved with urethral dilations. The patient is still alive and well.

Case \#3 - J.A., 52 year-old white male, truck driver. Five years ago, the patient noticed a vegetative tumor mass of slow growth in the scrotum, which ulcerated and did not heal. In May 2004, the patient was submitted to excisional biopsy of the lesion, which measured $4.0 \times 2.5 \times 2.5 \mathrm{~cm}$. The result demonstrated a well-differentiated infiltrating squamous cell carcinoma (grade-I) with verrucous pattern. The staging exams did not show any metastasis. The patient is doing well, without signs of recurrence or dissemination.

Case \#4 - C.F.B., 89 year-old white male, retired farmer. Eighteen months prior to his first visit, the patient noticed a $3.0 \mathrm{~cm}$ ulcerated lesion in the left hemiscrotum, presenting tumoral aspect. The patient was submitted to incisional biopsy, and the result showed squamous cell carcinoma (grade-II). In April 2004, the patient was submitted to wide resection of the lesion $(4.5 \times 3.5 \times 2.9 \mathrm{~cm})$, with a 1.0 $\mathrm{cm}$ safety margin. The result was similar to that of the biopsy, with free margins. The patient missed the follow-up and returned only in August 2005, presenting left inguinal tumoral lymph nodes, $8.0 \mathrm{~cm}$ wide, without mobility, with possible deep invasion, and considered irresectable. The scrotal scar had a good aspect. The patient was referred to radiotherapy and chemotherapy. Since then he is seen on an outpatient basis.

Case \#5 - J.C.A., 51 year-old brown male, truck driver. Ten years prior to the initial visit, the patient noticed the beginning of verrucous lesions in the pubic and scrotal areas. The patient sought medical aid and was treated for condylomatosis with topical application of podophyllin for countless times. Most of the lesions disappeared, except for a scrotal lesion that continued progressing and, three months before evaluation, it had reached $8.0 \mathrm{~cm}$ and was ulcerated. In December 2004, the patient was submitted to resection of the left hemiscrotum and study of the inguinal sentinel lymph node through the dynamic lymphoscintigraphy technique by use of ${ }^{99 \mathrm{~m}} \mathrm{Tc}$ and patent blue dye. The result was grade-I verrucous carcinoma with free margins and the removed lymph node was negative. The patient is doing well, without signs of recurrence.

Case \#6 - A.A.S., 31 year-old male, clerk, HIV positive. Two years prior to the initial visit, he noticed a red flat lesion, somewhat squamous, in the right hemiscrotum. He sought medical aid and was treated with topical cortical therapy without success. 
The lesion developed, increased in size, and ulcerated. In July 2005, the patient was submitted to excisional biopsy that evidenced a low grade squamous cell carcinoma. The patient did not present evidences of inguinal lymph nodes enlargement and is seen on an outpatient basis.

\section{COMMENTS}

Being initially described over 250 years ago and soon after, associated to the contact with the soot regarding chimney cleaners, scrotal neoplasia is considered the first occupational neoplasia recorded in medical literature $(1,2)$. Today, it is a known fact that the responsible agent for these cases of neoplasia is the carcinogen 3:4-benzpyrene, a hydrocarbon found in coal (5). The disease has some defined iatrogenic causes in its genesis: the Fowler's solution, an arsenic composition which has been used to treat psoriasis in the past; the association with psoralene and ultraviolet A radiation (PUVA) also employed in the treatment of this disease, causing solar keratosis and epidermoid dysplasia $(6,7)$; and the radiotherapy used in the treatment of scrotal eczema or groin lymphoma (8). Besides the role of hygiene as a probable cause, mechanical or chemical irritation is also questioned because there have already been cases reported in carriers of hypospadia, the scar of Fournier's gangrene, and spinal cord injury with urinary incontinence and chronic use of rubber urinals (9-11). From the total of patients, three were truck drivers, professionals who do not always practice the ideal type of hygiene, besides being exposed to diesel exhaust and having mechanical attrition of the scrotal area. In these workers, there are significant positive trends in lung cancer risk with increasing cumulative exposure to diesel exhaust (12). High risks have also been reported for other sites: skin, larynx, bladder, and kidney (12). There has been recorded a little higher incidence of scrotal carcinoma in the Iranian nomad (old Persia) population, who used to carry bags containing embers of coal underneath their clothes to keep them warm in the winter. Another issue to be raised is the role of HPV viruses, especially HPV16 and HPV18, in the genesis of a less aggressive variant, the verrucous carcinoma. These viruses are the same ones related to penis cancer (8). Likewise, truck drivers are traditionally considered one of the most sexual promiscuous groups in Brazil (13).

In the genesis of basal cell carcinoma, which corresponds to $5 \%$ of scrotal neoplasia, the etiology in question is immunosuppression due to aging, UV rays used in other sites, and the previous use of radiotherapy.

The natural history of scrotum cancer seems to be very similar to that of the penis and the protocols applied to the latter can be applied to the former (1).

Clinically, the lesion is usually presented isolatedly in the 6th decade of life, with slow growth, ulcerating after six months. Since it takes patients from eight to twelve months to seek medical help, a biopsy of the scrotum should be performed whenever suspicious growth is present (1).

The differential diagnosis should be chosen between squamous cell carcinoma, which is the most common lesion, and other neoplasias, such as malignant melanoma, reticular cell sarcoma, rhabdomyosarcoma, leiomyosarcoma, liposarcoma, basal cell carcinoma, extra mammary Paget's disease, Bowen's disease (in situ carcinoma), epithelial dysplasia and epithelioid sarcoma (14); benign lesions should also be taken into account: sebaceous cyst, acanthoma, hemangioma, leiomyoma, lymphangioma, fibroma, lipoma, myxoma, pigmented nevus, syphilis, psoriasis, eczema, periurethral abscess, tuberculous epididymitis and cutaneous schistosomiasis (15). Scrotal metastatic neoplasias are also uncommon and they have already been recorded as originating from the lungs, kidneys, ureter, bladder, appendix, and colon (16-20).

The preferred diagnostic method is the excisional or incisional biopsy, depending on the extension of the neoplasia.

The staging follows the basic principles of penis neoplasia staging: physical exam describing the extension and depth of the lesion, palpation of inguinal lymph nodes, pelvis imaging exams (CT or MRI) to evaluate pelvic lymph nodes and thorax X-ray to evaluate the lungs. There are records of dynamic scintigraphy (study of the sentinel lymph node) with use of ${ }^{99 \mathrm{~m}} \mathrm{Tc}$ and the patent blue dye, similar to the method described for penis cancer (21). In cases \#5 and \#6 described here, we used this technique, and it 
was possible to remove one inguinal lymph node ipsilateral to the scrotal lesion.

When treating the primary lesion, the intervention must be fast, just like it happens in penis cancer. The reason is that the survival rate is low if the disease progresses, with $30 \%$ of deaths happening soon after the progression (22). This is what has happened in case \#1 of the present series.

The excision with a surgical margin of $2 \mathrm{~cm}$ is recommended, followed by primary closing of the incision or use of grafts or flaps if the wound is large. Testicles should be preserved whenever possible by maintaining them in its own hemiscrotum, or transferring them to the contralateral hemiscrotum (23). Whenever this procedure is not feasible, testicles should be buried in the thigh subcutaneous tissue or protected with musculoskeletal flaps. If there the testis is affected, inguinal radical orchiectomy should be performed, similar to the treatment given to primary testicular tumor (1).

Inguinal lymphadenectomy or prophylactic inguinal iliac lymphadenectomy, for non-palpable lymph nodes, is controversial and it should be kept for palpable tumors after the use of antibiotic therapy, a protocol that is also similar to that of penis carcinoma (1). Lymphadenectomy should be bilateral, since the superficial lymph vessels of the scrotum communicate freely. In the presence of pelvic invasion, the prognosis has been poor. Simplified inguinal lymphadenectomy, with the preservation of the saphena vein, should be the method of choice (1).

Radiotherapy can also be applied, especially for verrucous carcinoma or for patients who do not accept surgery. In one case report, a 6200 cGy dose, in 31 fractions, allowed local control and significant reduction of symptoms (24). In another report, radiotherapy was given as initial treatment to 9 of 65 cases and showed no increment to survival rate after adjustment for other variables (22).

Reports on the treatment of systemic disease are scarce. BEP is the scheme that is applied most often, being also similar to what happens on penis cancer $(22,25)$.

The most important predictors of survival are stage and age at diagnosis. Survival rates varies progressively with combinations of these two variables regarding subjects younger than 65 years old and seen at diagnosis presenting a 5 years survival rate of $75 \%$ or more, compared with $17 \%$ for subjects who are 65 years old and older with regional or distant spread (22).

Contrary to what happens to most neoplasias, scrotal carcinoma seems to be heading for extinction. Most cases have been reported during the first half of the last century, and, nowadays, they are reported in an anecdotal way (2). If such forecast does not come true, it should be established, among other things, if the HPV virus has any relationship in the genesis of such neoplasia, in addition to accomplishing genomic study in the few described cases, and to confirming the role of the sentinel lymph node study with the use of the dynamic lymphoscintigraphy technique.

\section{CONFLICT OF INTEREST}

None declared.

\section{REFERENCES}

1. Chodak GW: Carcinoma of the Scrotum. In: Graham SD, Keane TE, Glenn JF (eds.), Glenn's Urologic Surgery. Philadelphia, J.B. Lippincott Co. 1991; pp. 90713.

2. Graves RC, Flo S: Carcinoma of the scrotum. J Urol. 1940; 43: 309-32.

3. Ray B, Whitmore WF Jr.: Experience with carcinoma of the scrotum. J Urol. 1977; 117: 741-5.

4. Souto CA, Lisboa JF, Telöken C, Basse RF, Lugli AC, Fernandes AM: [Scrotal neoplasia: a case report]. J Bras Urol. 1988; 14: 338-9. (in Portuguese).

5. Bostrom C, Gerde P, Hanberg A, Jernstrom B, Johansson C, Kyrklund T, et al.: Cancer risk assessment, indicators and guidelines for polycyclic aromatic hydrocarbons in the ambient air. Environ Health Perspect. 2002; 110: 451-89.

6. Stern RS: Genital tumors among men with psoriasis exposed to psoralens and ultraviolet $\mathrm{A}$ radiation (PUVA) and ultraviolet $\mathrm{B}$ radiation. The Photochemotherapy Follow-up Study. N Engl J Med. 1990; 322: 1093-7.

7. Orihuela E, Tyring SK, Pow-Sang M, Dozier S, Cirelli $\mathrm{R}$, Arany I, et al.: Development of human papillomavirus type 16 associated squamous cell 
carcinoma of the scrotum in a patient with Darier's disease treated with systemic isotretinoin. J Urol. 1995; 153: 1940-3.

8. Burmer GC, True LD, Krieger JN: Squamous cell carcinoma of the scrotum associated with human papillomaviruses. J Urol. 1993; 149: 374-7.

9. Pomara G, Pomara S, Travaglini F, Maras L, Selli C: Verrucous scrotal carcinoma in a patient with hypospadias: is there a possible association? Urology. 2003; 61:224.

10. Chintamani, Shankar M, Singhal V, Singh JP, Bansal A, Saxena S: Squamous cell carcinoma developing in the scar of Fournier's gangrene-case report. BMC Cancer. 2004; 4: 16.

11. Grundy D, Jones AC, Powley PH: Carcinoma of the scrotum associated with rubber urinals. Case report. Paraplegia. 1993; 31: 616-7.

12. Boffetta P, Jourenkova N, Gustavsson P: Cancer risk from occupational and environmental exposure to polycyclic aromatic hydrocarbons. Cancer Causes Control. 1997; 8: 444-72.

13. Lacerda R, Gravato N, McFarland W, Rutherford G, Iskrant K, Stall R, et al.: Truck drivers in Brazil: prevalence of HIV and other sexually transmitted diseases, risk behavior and potential for spread of infection. AIDS. 1997; 11 Suppl 1: S15-9.

14. Onol FF, Tanidir Y, Kotiloglu E, Bayramicli M, Turhal S, Turkeri LN: Proximal type epithelioid sarcoma of the scrotum: a source of diagnostic confusion that needs immediate attention. Eur Urol. 2006; 49: 406-7.

15. Joary P, Neiva A: Schistosomose genital: rara localizaçäo. J Bras Urol. 1980; 6: 289-90.
16. Dogra V, Saad W, Rubens DJ: Sonographic appearance of scrotal wall metastases from lung adenocarcinoma. AJR Am J Roentgenol. 2002; 179: 1647-8.

17. Aridogan IA, Satar N, Doran E, Tansug MZ: Scrotal skin metastases of renal cell carcinoma: a case report. Acta Chir Belg. 2004; 104: 599-600.

18. Iiyama $\mathrm{T}$, Watanabe $\mathrm{H}$ : Scrotal metastasis from ureteral cancer. J Urol. 2001; 165: 1637.

19. Saito S: Solitary cutaneous metastasis of superficial bladder cancer. Urol Int. 1998; 61: 126-7.

20. Niesel T, Bohm J, Paul R, Breul J, Hartung R: Rare metastases of signet ring cell carcinomas to the scrotum: report of two cases. Urology. 1996; 47: 76971.

21. Greene TD, Abrahams HM, Moran ME: Dynamic lymphoscintigraphy for staging scrotal carcinoma. J Urol. 2001; 165: 536-7.

22. Roush GC, Fischer DB, Flannery JT: A populationbased study of survival after scrotal carcinoma. Cancer. 1985; 55: 666-71.

23. Arango O, Bielsa O, Lorente JA, De Leon E, Mas AG: Hemiscrotectomy with contralateral testicular transposition for scrotal cancer. J Urol. 2002; 168: 14067.

24. Foroudi F, Turner S: Verrucous scrotal carcinoma: a radioresponsive tumor. J Urol. 1999; 162: 1694-5.

25. Arai Y, Kinouchi T, Kuroda M, Usami M, Kotake T: A case of scrotal cancer with inguinal lymph node metastasis treated by multidisciplinary modalities including chemotherapy with methotrexate, bleomycin and cisplatin. Hinyokika Kiyo. 1997; 43: 683-5.

\section{Correspondence address:}

Dr. Daniel Seabra

Rua Brasil, 1500, America

Barretos, Sao Paulo, 14783-180, Brazil

E-mail: daniel.seabra@terra.com.br 


\section{EDITORIAL COMMENT}

Primary malignant lesions of the scrotum are rare albeit they pose a particular concern for the urologist in terms of both diagnosis and management. There is a wide range of differential diagnosis and predisposing factors. In the present study, the authors raise the question of increased tendency of truck drivers towards developing these lesions, which is attributable to chemical exposure and risk of HPV infection. As indicated in this paper, the patients were admit at various stages of the disease and generally

\section{EDITORIAL COMMENT}

The authors have presented a retrospective review of six cases of scrotal carcinoma over a decade. While the increased risk of scrotal cancer in chimney sweepers is well established, it is not specifically linked to a particular occupation in the modern era.

The health hazards of diesel exhaust have been highlighted before, but mostly in relation to lung cancer. Diesel exhaust is considered a probable human carcinogen by the International Agency for Research there is a long interval between the onset of the lesion and primary admission. Correct diagnosis with the aid of immunohistochemical studies and sufficient clinical staging followed by prompt management is of utmost importance. Radical resection is the mainstay of the treatment, and although the prognosis is generally poor, a subset of patients with advanced disease may achieve potential cure with systemic chemotherapy and/or radiotherapy.

Dr. Fikret Fatih Onol Section of Urology Sakarya Training \& Research Hospital Sakarya, Turkey E-mail:ffonol@yahoo.com

on Cancer. This was based on the occurrence of lung cancer among truck drivers, bus drivers and railroad workers, who are exposed to diesel exhaust.

The authors have raised an interesting question, regarding increased risk of scrotal cancer in truck drivers. The number of cases in this series is small. As scrotal cancer is rare now, it will require multi-center analysis of a large number of cases, to gain more information on this hypothesis.

Dr. T. John

Department of Urology

Wayne State University

4160 John $R$

Detroit, MI 48201, USA

E-mail:tonytjohn@yahoo.com 